\title{
APPLICATION OF IMPROVED BP NEURAL NETWORK USING GENETIC ALGORITHM IN FAULT ASSESSMENT OF BEARING IN ELECTROMECHANICAL SYSTEM
}

\author{
Shuyue Zhang \\ Guangzhou College of Technology and Business, Guangzhou, 510850, Guangdong, China. \\ Email: zhangshuyue18@yeah.net
}

\begin{abstract}
The research aims at the serious economic loss and life-threatening problems caused by mechanical and electrical system accidents and bearing failures. The fault detection and identification of bearings in mechanical and electrical systems are discussed. The structure, fault cause, vibration mechanism, and characteristic frequency of rolling bearing are analyzed, and the noise of vibration signals is removed and eliminated in light of the characteristics of the initial fault signal of rolling bearing. Because of the shortcomings of the wavelet, wavelet packet transform is proposed to characterize the normal state of rolling bearing, rolling element fault, inner ring fault, and outer ring fault signal. Based on the characteristics of global optimization of GA (genetic algorithm), the algorithm falls into local optimal value following the defects of BP (backpropagation) neural network and uses GA to optimize the BP neural network algorithm for fault diagnosis of rolling bearings. According to the experimental results, when the evolution algebra of the fault diagnosis model GA-BP is 8 at the drive end, the optimal classification accuracy of the population reaches $98.83 \%$. In this case, a rolling element fault in the test data is misclassified. When the evolution algebra of the GA-BP fault diagnosis model is 2 at the fan side, the overall optimal classification accuracy reaches $97.62 \%$ in total. Under this condition, a rolling element fault and an outer ring fault are misclassified in the test data. Through the comparison experiment with the traditional optimized BP neural network, it is found that the GA-BP neural network algorithm model is suitable for the fault classification of rolling bearings.
\end{abstract}

Keywords: Genetic Algorithm; BP neural algorithm; Bearing Fault Assessment; Electromechanical System.

\section{Introduction}

With the continuous development of science and technology, the development of modern industry tends to expand towards intelligent integration. As a tool of electric energy conversion in industrialization, rotating electromechanical devices become more and more important in various fields. Among them, the bearing is one of the important components of rotating electromechanical equipment, supporting the spindle and transmitting torque of electrical equipment [1]. Good performance of bearings has a significant impact on the production and equipment operation. However, because of the operating conditions of motor bearings and their role in rotating equipment, motor bearings are very vulnerable to damage. Besides, due to the complex working environment of the electromechanical system, all kinds of faults will inevitably occur. If there is an unexpected major fault, it will not only cause huge economic losses, but also endanger people's lives, and even serious social consequences [2]. In industrial production, most bearings operate in complex and harsh environments, such as fatigue, environmental mechanical vibration, overload, bearing asymmetry, pollution, current slot, corrosion, improper lubrication, which results in motor failure in the bearing fault. This usually occurs in many cases [3]. Some studies show that the number of bearing failures is more than $40 \%$ of all motor failure events. Hence, in the field of motor fault diagnosis, the research of bearing fault diagnosis attracts the attention of many scholars [4]. After the economic development and the safety of industrial production are considered, the early fault of bearing should be diagnosed, the workload of fault motor should be adjusted in a planned way, and the spare motor can ensure normal industrial production [5]. This not only avoids more serious damage to the motor, but also reduces the human, material, and financial losses caused by bearing failure.

Mechanical vibration signal is widely used in equipment fault diagnosis because of its mature 
theory, intuitive reflection, and excellent diagnostic effect [6]. The vibration signal extracted from the sensor is the original signal, which contains a great deal of fault information with a certain amount of noise. Separating useful fault signals from original signals and extracting useful signal features are key to fault diagnosis [7]. At present, many scholars in China and foreign countries carry out a lot of research in the field of directional feature extraction and type recognition and achieve some breakthroughs. However, most of the current studies focus on denoising and feature extraction of bearing fault signals, and then a variety of methods are used to classify and identify them [8]. Although the research obtains much useful information, the process is too complex and time-consuming to achieve the expected results [9]. Nowadays, the continuous development of deep learning theory based on machine learning replaces machine learning and is widely used in various fields [10]. A deep network is constructed to extract the features of the input sample parameters layer by layer, and the bearing failure features are obtained by setting a nonlinear activation function for each layer of the network, thus eliminating the dependence on manual operation.

The motor bearing is taken as the main research object, and a large number of bearing vibration data are taken as the training sample. Afterward, the deep learning algorithm is applied to the bearing fault diagnosis to carry on the corresponding research.

\section{Bearing Fault Research Ideas and Experimental Design}

\subsection{Bearing Structure, Fault Type, and Diagnosis Method}

The bearing fault is one of the main types of motor fault and searching for effective and reliable methods to predict it is a hot topic that most scholars are interested in.
Given the complexity and particularity of the working environment, it is impossible to directly observe the size of the small fault points in the bearing. Therefore, the main studies of bearing fault diagnosis center on the analysis of bearing fault type caused by vibration [11]. The machine learning algorithm is more and more widely used in bearing fault classification with its development. The application of machine learning algorithm in bearing fault diagnosis includes three main parts: fault feature extraction, feature selection and fault classification. The feature extraction of azimuth tomography mainly refers to the extraction and subsequent analysis of vibration signal features. Nowadays, the commonly used methods for preprocessing bearing tomography X-ray vibration signals include envelope analysis, spectrum crossover, wavelet transform, EMD (Empirical Mode Decomposition), VMD (Variational Mode Decomposition). After the features are extracted by adopting the above method, the training samples used as classification algorithms are applied to select the type of training classification [12].

Figure 1 shows the structure of the rolling bearing. It consists of an outer ring, a rolling element (ball body), an inner ring, and a cage [13]. In general, the outer ring is connected to the housing of the bearing seat and the bearing box, keeping fixed or relatively fixed, which plays a role in supporting rolling elements. The inner ring of the bearing is usually connected to the journal and rotates with it together. The rolling elements mainly include spherical roller, cylindrical roller, tapered roller, and needle roller. They are evenly distributed in the raceway formed by the inner ring and the outer ring to reduce the friction between the inner ring and the outer ring by transferring the track sliding frication. The cage mainly keeps each rolling element at an appropriate distance to prevent collision and shedding between rolling elements.

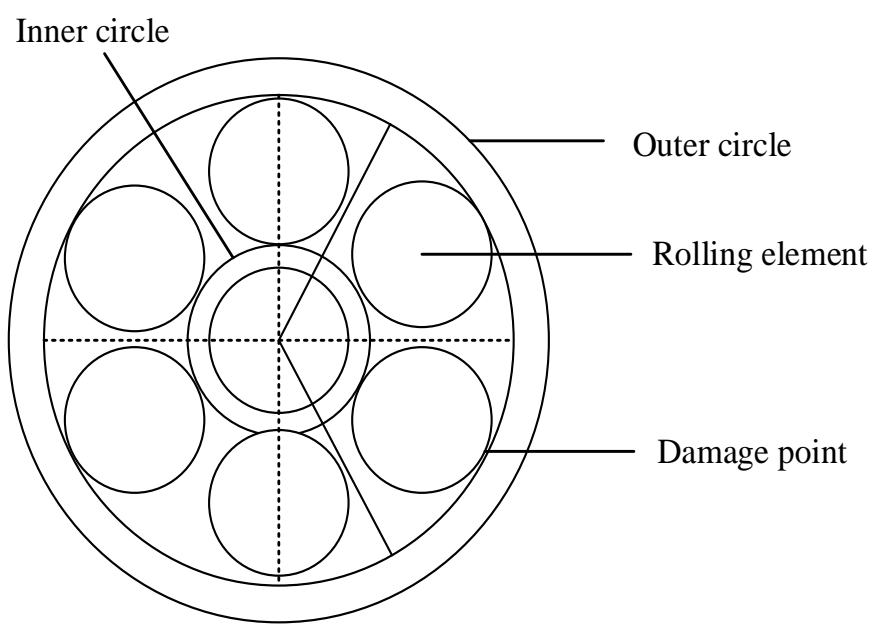

Figure 1. Structure of rolling bearing 
Improper installation of bearings and the interior materials may cause the failure of rolling bearings as well. Besides, continuous overload operation, corrosion of acid and alkali or foreign particles may lead to the abnormal state of bearings [14]. Even if the bearing is made of superior material and installed properly, the rolling bearing appears to wear, gap, or fatigue delamination in normal use under standard operation. Failure types of rolling bearings are mainly caused by fatigue spalling, wear, fracture, corrosion, adhesion, and indentation [15]. The fault analysis methods of rolling bearing mainly include vibration analysis, oil analysis, temperature detection, and acoustic emission. At present, the vibration analysis method is the most widely used fault analysis method [16]. There are usually two reasons for rolling bearing vibration, one is internal factors, such as the structural characteristics of the bearing itself, machining and assembly errors, and the failures during operation; the other is external factors like driving shaft motion. In practical diagnosis, vibration signals are usually obtained by sensors installed in the bearing seat or other positions. The sensor contains information generated by the combined action of internal and external factors [17]. Therefore, it is important to extract the vibration characteristics caused by the fault from the total vibration in the fault diagnosis of rolling bearings.

\subsection{Fault Characteristic Frequency of Rolling Bearing}

When the rolling bearing runs for a long time, the rolling bearing usually has various faults due to different degrees of damage to the surface of the internal assembly. When the bearing is running, the damage point will repeatedly and quickly collide with the surface of other parts, causing periodic vibration [18]. The natural frequency is generated by the impact vibration of the rolling element and the outer ring or inner ring and is related to the elasticity of the bearing, which is determined by the stiffness and mass of the rolling bearing. For various bearing assemblies, the natural frequency has a limited range.

Under normal operating conditions, the natural frequency $f_{n b}$ of the rolling element is:

$$
f_{n b}=\frac{0.848}{d} \sqrt{\frac{E}{2 \rho}}
$$

$d$ is the diameter of the rolling element measured in $\mathrm{m}$; $E$ is the elastic modulus of the rolling element material measured in $\mathrm{N} / \mathrm{m}^{2}, \rho$ represents the material density measured in $\mathrm{kg} / \mathrm{m}^{3}$.
The natural vibration frequencies of the bearing inner and outer rings are as follows:

$$
f_{n r}=\frac{n\left(n^{2}-1\right)}{2 \pi(D / 2)^{2} \sqrt{n^{2}+1}} \sqrt{\frac{E I}{M}}
$$

$D$ is the diameter of the neutral axis of the ring cross-section measured in $\mathrm{m}$; $n$ is the deformation wave number (vibration order), and $M$ is the mass of the unit length of the circle with the measurement of $\mathrm{kg} / \mathrm{m} . \mathrm{I}$ is the moment of inertia of the outer ring section around the neutral axis, and its unit is $m^{4}$. When the fault signal of the rolling bearing is analyzed, the working state of the rolling bearing can be identified by the characteristic frequency variation law of the inner ring, outer ring, rolling element, and cage of the rolling bearing.

When the rolling bearing works normally, the equation for calculating the rotation speed $v_{i}$ of a point on the inner ring track is as follows:

$$
v_{i}=2 \pi r_{1} f_{i}=\pi f_{i}(D-d \cos \alpha)
$$

$f_{i}$ is the rotation frequency of the inner ring raceway; $r_{1}$ is the radius of the inner ring raceway, $D$ is the diameter of the raceway, $d$ is the diameter of the rolling body, and $\alpha$ is the contact angle. The angle is formed by the contact point of the inner ring and the outer ring with the radial surface of the rolling element and bearing.

The equation for calculating the speed of the outer raceway is as follows:

$$
v_{o}=2 \pi r_{2} f_{o}=\pi f_{o}(D+d \cos \alpha)
$$

$f_{o}$ is the outer ring raceway rotation frequency and $r_{2}$ is the radius of the outer ring raceway.

The equation for calculating the rotational speed $v_{c}$ of the cage is as follows:

$$
v_{c}=\frac{1}{2}\left(v_{o}+v_{i}\right)=\pi f_{c} D
$$

The above equation for calculating the rotation frequency $f_{c}$ of the cage can be deduced as follows:

$$
f_{c}=\frac{1}{2}\left[\left(1+\frac{d}{D} \cos \alpha\right) f_{o}+\left(1-\frac{d}{D} \cos \alpha\right) f_{i}\right]
$$

The frequency $f_{o c}$ of a single rolling body on the outer raceway is: 


$$
f_{o c}=f_{c}-f_{o}=\frac{1}{2}\left(f_{i}-f_{o}\right)\left(1-\frac{d}{D} \cos \alpha\right)
$$

The rotation frequency $f_{b c}$ of the rolling body relative to the cage is:

$$
f_{b c}=\frac{1}{2} \times \frac{D}{d}\left(f_{i}-f_{o}\right)\left(1-\frac{d^{2} \cos ^{2} \alpha}{D^{2}}\right)
$$

When a defect or fault occurs in the inner ring, the equation for calculating the characteristic fault frequency $z f_{i c}$ of $z$ rolling bodies passing through a point on the inner ring trajectory is as follows:

$$
z f_{i c}=\frac{1}{2} z f_{i}\left(1+\frac{d}{D} \cos \alpha\right)
$$

When a defect or fault occurs in the outer ring, the equation for calculating the characteristic fault frequency $z f_{o c}$ of $z$ rolling bodies passing through a point on the inner ring trajectory is as follows:

$$
z f_{o c}=\frac{1}{2} z f_{i}\left(1-\frac{d}{D} \cos \alpha\right)
$$

When the rolling body has defects or faults, the equation of fault characteristic frequency $f_{b c}$ through the outer ring or inner ring is as follows:

$$
f_{b c}=\frac{D}{d} f_{i}\left(1-\frac{d^{2} \cos ^{2} \alpha}{D^{2}}\right)
$$

\subsection{Fault Feature Extraction of Motor Bearing}

The current motor bearing fault diagnosis algorithm includes three main parts: fault feature extraction, feature selection and fault classification.

At present, the key to realizing the current bearing fault diagnosis algorithm model is to extract the bearing vibration signal accurately, and the classification model is used to achieve the fault classification [19].
Bearing failure feature extraction is mainly used to extract and analyze the failure characteristics of vibration signals. However, due to the influence of electromagnetic interference and the environment, the collected bearing failure signals are affected by various noises. Therefore, the general algorithm is used to extract reliable and effective fault features for subsequent fault analysis. At present, the most commonly used data feature extraction method is Wavelet Packet Decomposition.

Wavelet analysis theory is developed based on Fourier transform, the algorithm of which cannot effectively extract the feature of obstacles contained in the signal when dealing with discrete signals [20]. Wavelet analysis breaks the limitation of the traditional time domain to some extent, and the processing effect for the discrete signal is better. By using the wavelet analysis algorithm, the collected signal is decomposed and reconstructed, which is more effective than the original data signal [21]. Wavelet packet decomposition is an improved wavelet analysis technology, which is suitable for multi-resolution analysis and processing applications. Wavelet analysis can only decompose the low-frequency component in the process of processing the signal.

Therefore, this decomposition method cannot better reflect the property of the features contained in the original signal [22]. Wavelet packet decomposition can segment signals in more detail at different levels. This solves the disadvantage that high-frequency components cannot decompose in the process of wavelet decomposition. Wavelet packet decomposition better shows the information contained in the localization time and the frequency of the signal, which is obtained through the multiscale decomposition of the signal frequency band and the "local refinement" of the high-frequency part.

Figure 2 is a schematic diagram of wavelet packet decomposition for the signal with 3 layers. The principle and realization of wavelet packet decomposition are as follows:

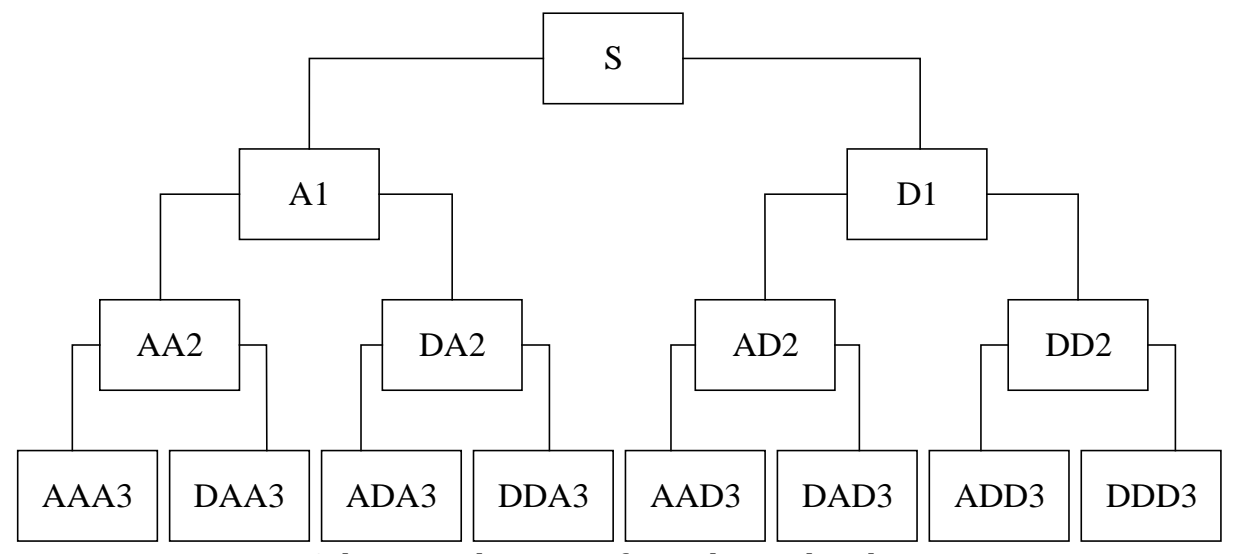

Figure 2. Schematic diagram of wavelet packet decomposition 
The relation equation of each subband waveform after decomposition is expressed as:

$$
S=A A A_{3}+D A A_{3}+A D A_{3}+D D A_{3}+A A D_{3}+D A D_{3}+A D D_{3}+D D D_{3}
$$

Suppose $U_{j}^{n}$ is a closed subspace of the function $U_{n}(t)$, and $U_{j}^{2 n}$ is defined as a closed space of the function $u_{2 n}(t) \cdot u_{n}(t)$ should satisfy the following two-scale equations:

$$
\begin{aligned}
& u_{2 n}(t)=\sqrt{2} \sum_{k \in Z} h(k) u_{n}(2 t-k) \\
& u_{2 n+1}(t)=\sqrt{2} \sum_{k \in Z} g(k) u_{n}(2 t-k) \\
& g(k)=(-1)^{k} h(1-k)
\end{aligned}
$$

In the above equations, $h(k)$ and $g(k)$ is orthogonal.

When $n=0$, the two-scale equation is as follows:

$$
\begin{aligned}
& u_{0}(t)=\sum_{k \in Z} h(k) u_{0}(2 t-k) \\
& u_{1}(t)=\sum_{k \in Z} \mathrm{~g}(k) u_{0}(2 t-k)
\end{aligned}
$$

After wavelet packet decomposition and reconstruction, wavelet packet can be used to deal with signals changing at any time and frequency.

Assuming $g_{j}^{n}(t) \in u_{j}^{n}, g_{j}^{n}(t)$ is known according to the two-scale equation:

$$
g_{j}^{n}(t)=\sum_{l} d_{l}^{j-n} u_{n}\left(2^{j} t-l\right)
$$

$\left\{d_{l}^{j, 2 n}\right\}$ and $\left\{d_{l}^{j, 2 n+1}\right\}$ are used to calculate $\left\{d_{l}^{j+1, n}\right\}$. And the reconstruction algorithm of the wavelet packet is as follows:

$$
d_{l}^{j+1, n}=\sum\left[h_{l-2 k} d_{l}^{j, 2 n}+g_{l-2 k} d_{l}^{j, 2 n+1}\right]
$$

The energy of the signal function can be expressed as:

$$
\|f(t)\|=\int_{-\infty}^{+\infty}|f(t)|^{2} d t
$$

According to the law of conservation of energy, the wavelet packet can decompose the nonoverlapping energy orthogonal to the adjacent frequency band. Therefore, the square of the coefficient obtained by decomposing wavelet packets is equal to the energy of the original signal in the time domain.

$$
E_{j}=\sum_{i=1}^{N}\left\|n_{i}\right\|^{2}
$$

$j$ is the corresponding frequency band after decomposition; $N$ is the number of data sampling points, and $n_{i}$ is the coefficient obtained after decomposition.

The feature vector composed of wavelet packet energy of the corresponding spectrum is used as the feature of rolling bearing fault diagnosis. The vibration signal of rolling bearing is unstable and nonlinear, and the energy characteristic changes with the change of working condition of rolling bearing. According to the analysis, the vibration signal of the three-layer rolling bearing with the wavelet packet is used. The normalized processing of waveform energy value is used as the feature vector of fault diagnosis, and the process is as follows:

(1) Noise is removed from vibration signals collected from rolling bearings based on wavelet transform.

(2) Eight different frequency band components are obtained by three-layer decomposition and reconstruction of noise-removed vibration signals based on wavelet packets.

(3) Calculate the energy values of the eight reconstructed waveforms and use them as feature vectors of the normalized rolling bearing fault signals. The normalized equation is:

$$
E_{i}=\frac{X_{i}}{\sum_{i=1}^{n} X_{i}}
$$

$X_{i}$ is the characteristic energy of each frequency band, and $E_{i}$ is the characteristic value after normalization. The range of $i$ goes $0,1,2, \ldots, 7$.

In the initial stage of rolling bearing fault diagnosis, the fault vibration signal is relatively weak and greatly affected by noise, like a noisy bearing running environment. Therefore, when the energy characteristics of the vibration signal are being extracted, the signal to remove the vibration signal must be used for preprocessing to eliminate the noise interference. Hence, the wavelet is proposed to denoise the collected vibration signals. 
The original simulation signals are created in MATLAB to verify the effectiveness of wavelet denoising. Meanwhile, the de-noised data are performed wavelet packet decomposition and feature extraction by using MATLAB.

\subsection{Research on Fault Diagnosis of Rolling Bearing based on BP}

The artificial neural network is a mathematical model, which simulates the function and structure of the biological neural network. BP neural network is a neural network model based on a single-layer neural network, and the training is carried out according to the error backpropagation algorithm [23]. BP neural network is a typical three-layer network model, and its specific structure is shown in Figure 3.

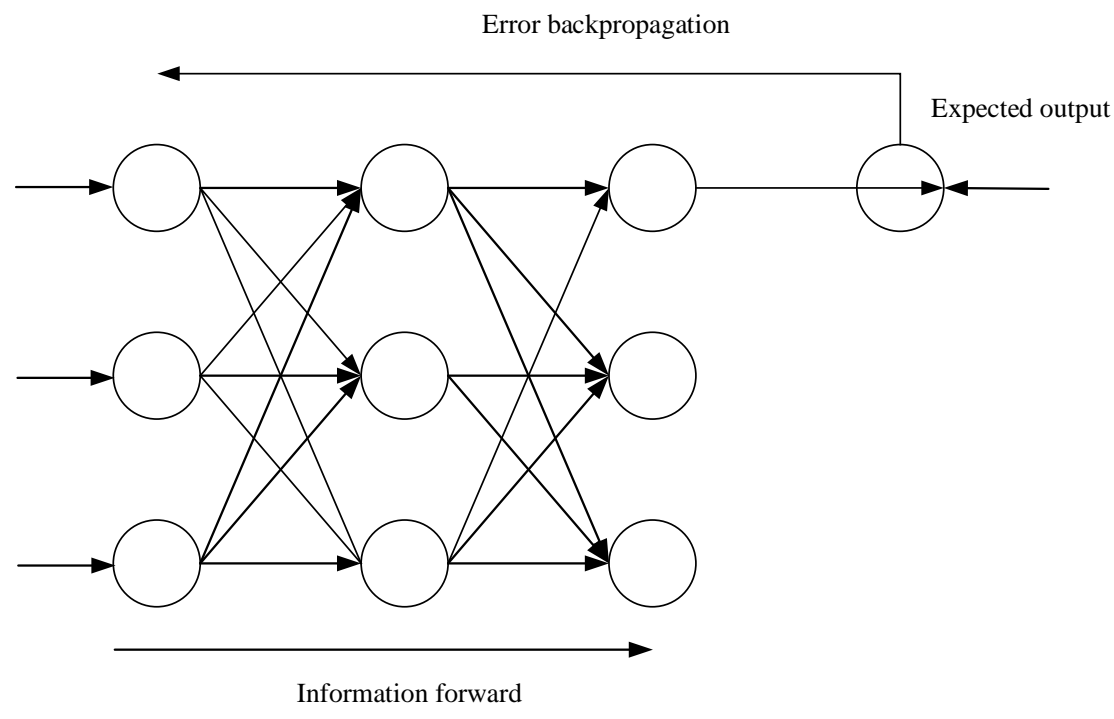

Figure 3. Structure of 3-layer BP neural network

BP neural network algorithm divides the training process into two stages.

The first stage is the forward dissemination of information. The input data calculates the actual output of each neuron based on the corresponding weights and thresholds. The mathematical model expression equation is as follows:

$$
\begin{aligned}
& y_{i}^{l}=f\left(x_{i}^{l}\right) \\
& x_{i}^{l}=\sum_{j=1}^{N_{l-1}} w_{i j}^{l} y_{j}^{l-1}+\theta_{i}^{l}
\end{aligned}
$$

$y_{i}^{l}$ is the output value of the $i$-th node in the $l$ layer; $x_{i}^{l}$ is the activation value of the $i$-th node in the $l$ layer; and $w_{i j}^{l}$ is the weight value of the $j$-th node in the $l-1$ layer. $\theta_{i}^{l}$ is the threshold value of the $i$-th node in the $l$ layer; $N_{l}$ is the number of nodes in the $l$ layer, $l$ is the total number of the layers, and $f$ is the neuron activation function.

The second stage is the error backpropagation stage. If the error between the output value and the expected value of the first stage does not meet the precision requirement and does not reach the number of the set iterations, the backpropagation process is carried out according to the steepest descent method. Change the weight of each connection in the direction of error reduction according to the error between the output value and the expected value. The expression equation of the process is as follows:

$$
\Delta w_{i j}=-\eta \frac{\partial E}{\partial w_{i j}}
$$

$\eta$ is the learning rate.

The weight adjustment equation is:

$$
w_{i j}(t+1)=w_{i j}(t)-\eta \frac{\partial E}{\partial w_{i j}}
$$

Algorithm steps for BP neural networks are as follows:

(1) Initialize algorithm parameters, such as determining the number of input layer nodes, hidden layer nodes, and output layer nodes.

(2) Calculate the hidden and output layers and use the weights and thresholds of the external input and hidden layers as the hidden layer outputs. The output of the output layer is calculated according to the output of the hidden layer and the weight and threshold of the hidden layer and the output layer.

(3) Calculate the loss values based on the actual and expected outputs of the network model to obtain model errors. 
(4) Update weights and thresholds.

(5) If the accuracy of the algorithm or the maximum number of iterations is not satisfied, return to step (2).

BP neural network has a strong nonlinear mapping ability. During the training, "learning content" is adaptively stored in the weights of BP neural networks. Therefore, BP neural network has high self-study ability. But the disadvantage of the BP neural network algorithm falls into extremes in the training process. Based on the BP neural network, 180 sets of four energy characteristic samples at the drive end and fan end of the motor are selected as training samples, the remaining 80 groups as test samples. Furthermore, the label is set according to the four states of the rolling bearing. A three-layer BP neural network model is established by using MATLAB. In the light of the input and output of the energy characteristic sample, the number of neurons in the input layer of the neural network is 8; the number of neurons in the output layer is 4 , and the number of neurons in the hidden layer is 17. Besides, the activation function and training function are Sigmoid. BP training algorithm uses variable learning rates as training targets, the training target is 0.0001 , the learning rate is 0.01 , and the training step is 3000 . Each of 720 sets of energy feature training samples from the drive and fan ends is input into the BP neural network for training, and then 80 sets of test samples are input into the trained fault diagnosis model for testing.

\subsection{Research on Fault Diagnosis of Rolling Bearing based on GA-BP}

A genetic algorithm is proposed by Holland in the United States. The algorithm is inspired by evolution, namely species selection and population genetics. It is an algorithm focused on process calculation. The potential solutions to problems in the form of chromosomes are analyzed by genetic algorithms. Fitness is an important function of a genetic algorithm, and its value is used to analyze the advantages and disadvantages of dyeing. During the process of optimizing the algorithm, each generation enters their living environment by gradually forming a new generation of potential solutions by using algorithms, selection, intersection, variation, and other operators, showing their strong adaptability [24]. GA has important functions, such as global solution space search, parallel processing, and wide adaptability. Global solution space search can avoid falling into local optimal solutions, and the search efficiency is high. Because in the operation of the algorithm, each individual of the independent variable is searched in parallel in the unit of population, and GA can run directly.
As a structure, it has a wide range of adaptability [25]. Thus, GA is widely used in many fields, such as adaptive control, image processing, production scheduling, and combinatorial optimization.

The basic steps of GA are as follows:

(1) Coding: The solution space of the problem is abstractly mapped to the structure of the gene string, and the solution space is usually encoded as a finite length binary string consisting of 0 and 1 .

(2) Initialization population: to form an initialization population, $\mathrm{N}$ individuals are generated in the possible problem-solving space.

(3) Calculation of fitness function values: fitness function values are indicators for the evaluation of chromosomes, and fitness values for each coding gene string are calculated based on fitness functions.

(4) Selection: The selection operation is based on the fitness value of step (3) and forms a new population by selecting excellent individuals.

(5) Cross: Randomly select the same location of the two gene strings and form a new individual through a combination of crosses.

(6) Mutations: The reversal of a specific position of a particular gene. The new individuals produced by the mutation are added to the parent generation. These changes mimic natural gene mutations.

Generally, when the population is small, the calculation speed of the genetic algorithm is considered to be faster. The number of the population is usually 200100, the crossover probability is usually $0.45-0.9$, the mutation probability is usually smaller, namely 0.001-0.01; and the final algebra is usually between 100-1000. The thresholds and weights of traditional BP neural network algorithms are usually generated randomly and tend to be classified as local optimal solutions. The GA global search function is applied to address this defect in traditional BP neural network algorithms and identify the four states of rolling bearings more accurately, Besides, GA can optimize BP neural network model to perform fault diagnosis of rolling bearings.

\section{Experimental Results Analysis and Discussion}

\subsection{Experiment on Fault Feature Extraction of Rolling Bearing based on Wavelet Packet Decomposition}

Fast Fourier operation is carried out on the time domain waveform with noise and denoising signal to ensure that the signal processed by wavelet denoising does not filter out the useful components of the original signal. Moreover, the fault feature extraction results of vibration signals based on wavelet packet decomposition are obtained by comparing the noisy and de-noised signals. 
The spectra and results are shown in Figure 4:
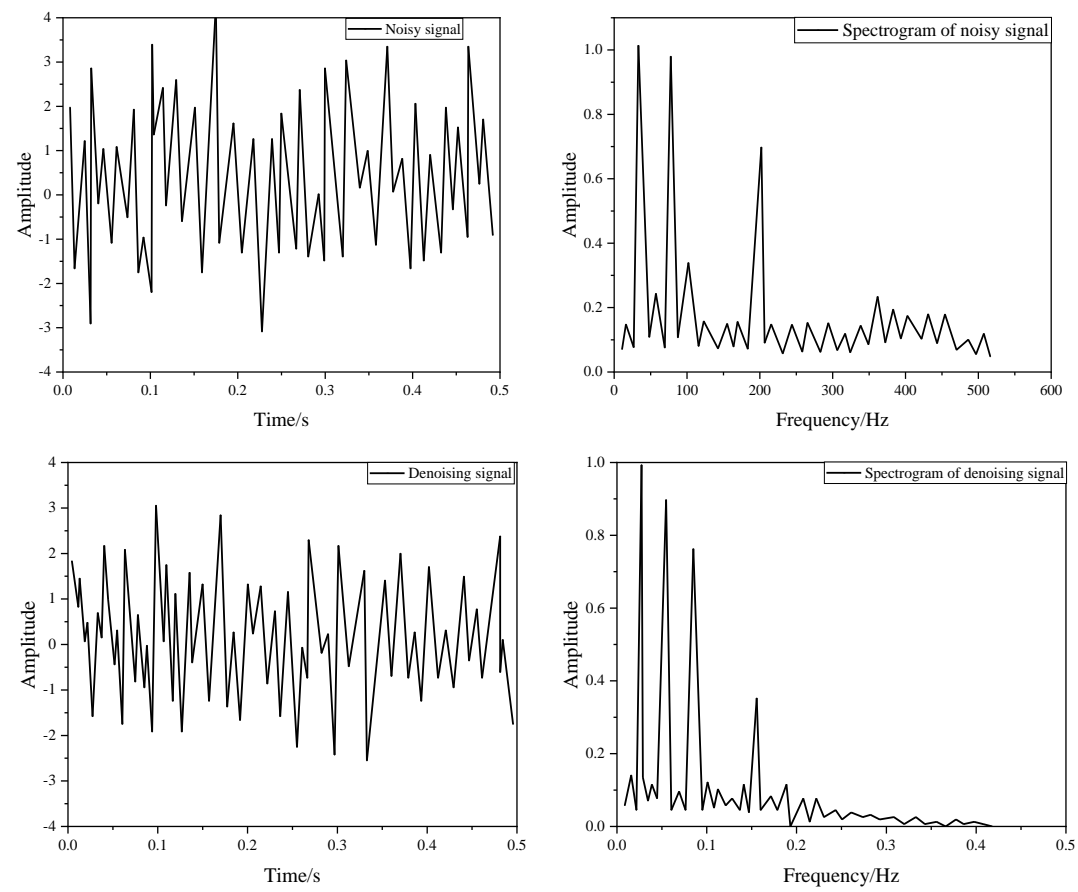

Figure 4. Waveform and spectrum of noisy and denoised signals

Figure 4 shows that the effective frequencies of $25 \mathrm{~Hz}, 50 \mathrm{~Hz}, 100 \mathrm{~Hz}$, and $180 \mathrm{~Hz}$ are retained after wavelet denoising, and the signal noise is effectively removed.
It can be concluded that wavelets can be used to deal with rolling bearing vibration signals to reduce noise interference and improve the signal-to-noise ratio.

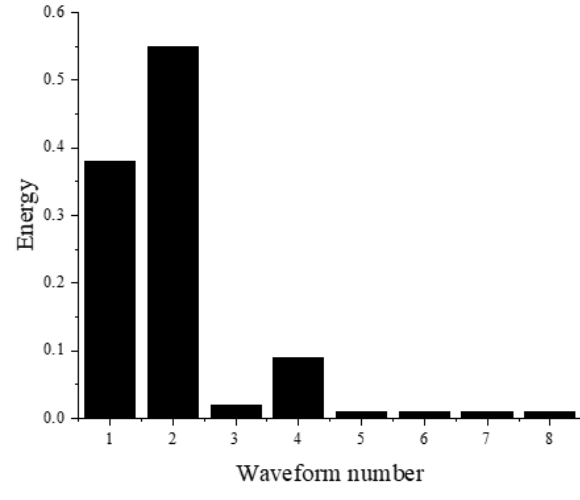

(1) Characteristics of normal bearing energy

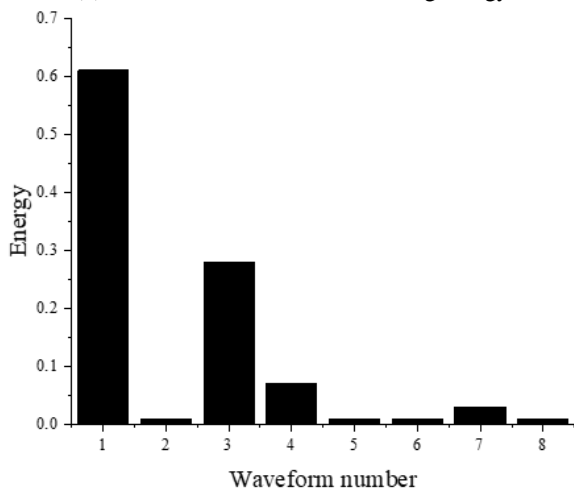

(3) Rolling failure energy characteristics

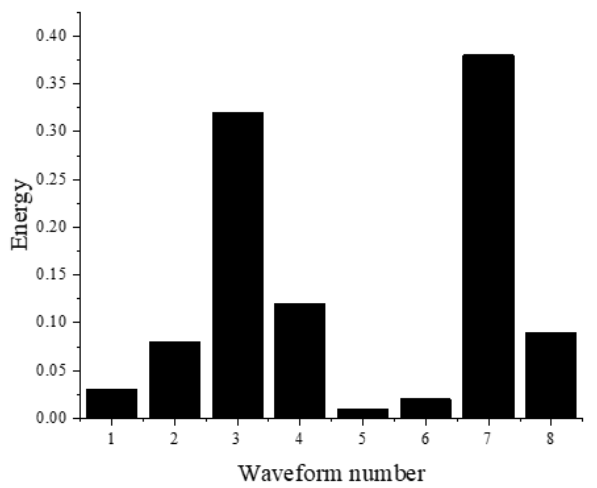

(2) Inner ring failure energy characteristics

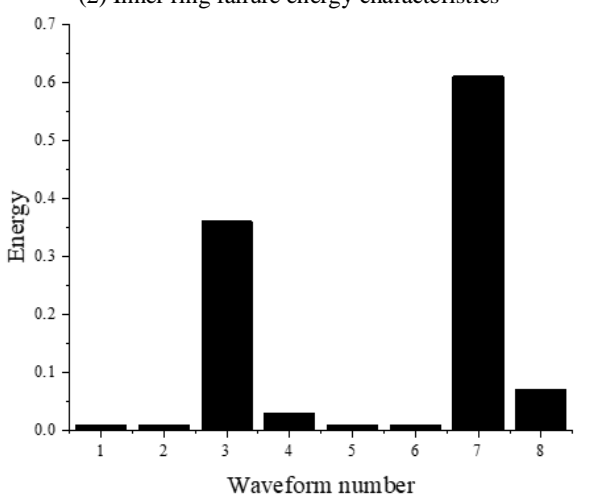

(4) Outer ring failure energy characteristics

Figure 5. Energy characteristic of four types at the drive end 


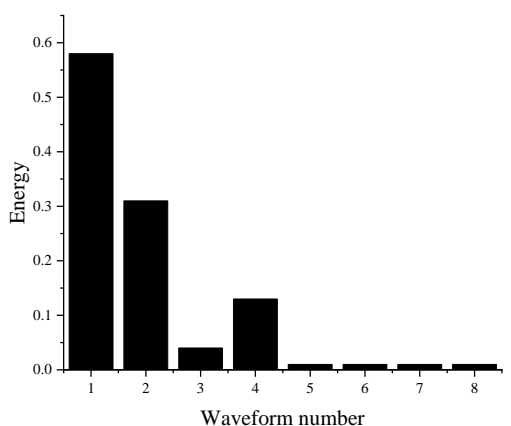

(1) Characteristics of normal bearing energy

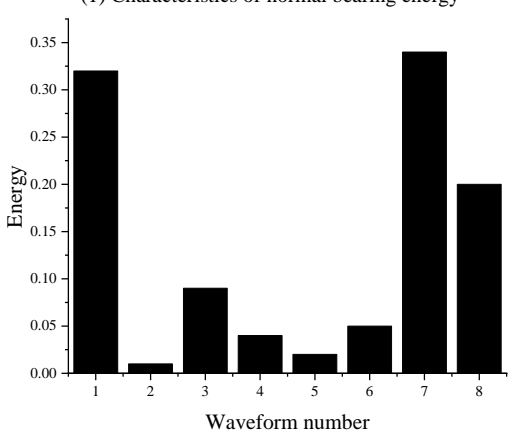

(3) Rolling failure energy characteristics

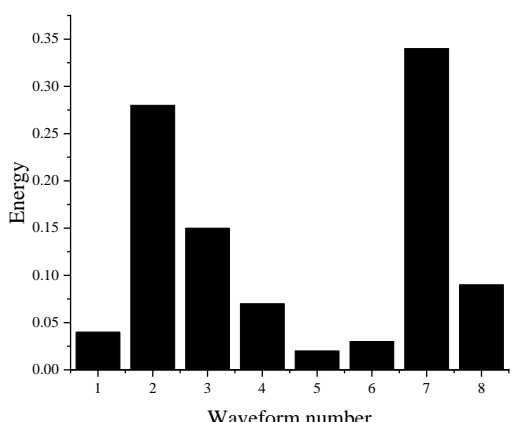

(2) Inner ring failure energy characteristics

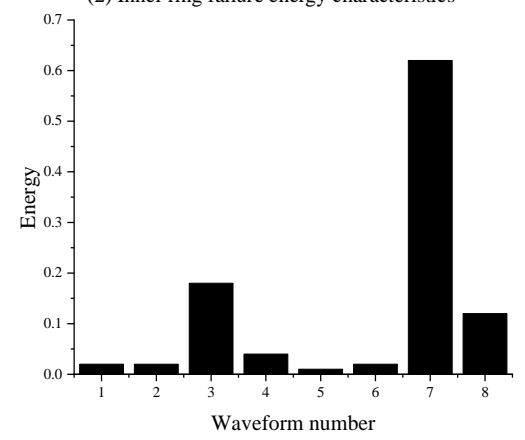

(4) Outer ring failure energy characteristics

Figure 6. Energy characteristics of four types at the fan end

The experimental results in Figure 5 and Figure 6 indicate the variation of the amplitude of the eightbar waveform of the normal state is quite different after the decomposition of the three-layer wavelet packet for the normal state. The inner ring fault, the rolling body fault, and the outer ring fault, which show that the data information they contain is different. After the wavelet packet decomposition of the three-layer, the energy characteristic distribution of the four bearing states is also different. Therefore, the characteristic samples can be constructed by extracting energy eigenvalues as the basis of bearing fault diagnosis.

\subsection{Experimental Results of Rolling Bearing Fault Diagnosis based on GA-BP}

720 sets of training samples and 80 sets of test samples are selected at the drive end and the fan end respectively, they match the data used in section 2.5 .
A rolling bearing vibration signal data set provided by the electrical engineering laboratory of CWRU (case west storage university) is used.

The genetic algorithm has a crossover probability of 0.4 , the mutation probability of 0.1 , and the classification accuracy as a fitness function. 720 sets of training samples are imported into the GA-BP model from the drive end and the fan end respectively.

Figure 7(1) shows the goodness-of-fit curve when the model at the driver side is deployed. 80 sets of test samples are imported into the trained GA -BP fault diagnosis model. Figure $7(2)$ is the GA-BP classification result of the driver end. Figure $8(1)$ shows the evolutionary goodness-of-fit curve of the fan-end model, and Figure 8(2) tells the classification results of the fan-end GA-BP model.

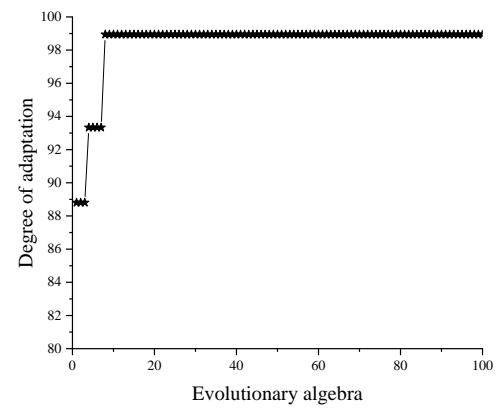

(1) Drive end fitness curve

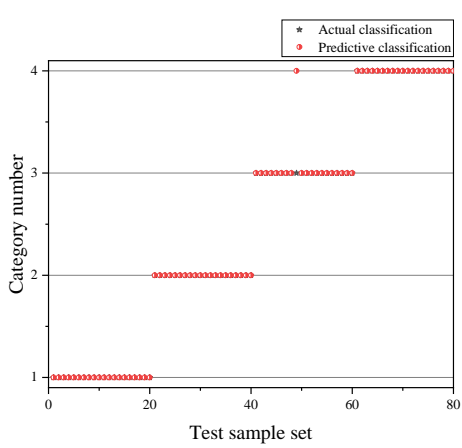

(2) The actual classification and prediction classification map of the test set

Figure 7. Experimental results at the drive 


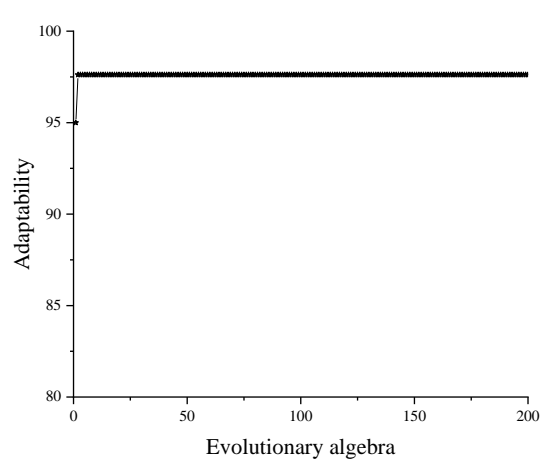

(1) Drive end fitness curve

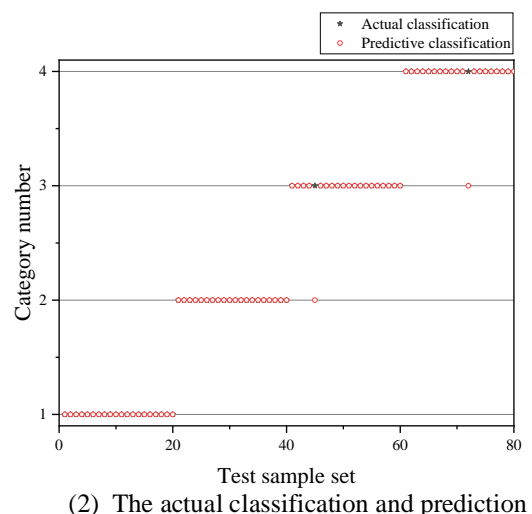

(2) The actual classification and prediction classification map of the test set

Figure 8. Experimental results at the fan

The experimental results reflect that when the evolution algebra of the fault diagnosis model GA-BP at the driving end is 8 , the optimal classification accuracy of the population reaches $98.83 \%$. In this case, a rolling element fault data classification error occurred in the test. When the evolution algebra of the fault diagnosis model at the fan end is 2, the overall optimal classification accuracy reaches 97.62\%. Under this condition, a rolling element fault and an outer ring fault are misclassified in the test data.
When BP neural network model and GA-BP neural network model are used for fault diagnosis, training samples and testing samples will be randomly selected to avoid the influence of different data on the classification results.

And the corresponding classification results are compared in the same experimental data.

Table 1 lists the classification results of BP neural network and GA-BP neural network models.

Table 1 BP \& GA-BP algorithm fault identification results

\begin{tabular}{ccccccc}
\hline Algorithm & Type & $\begin{array}{c}\text { Normal } \\
\text { state }\end{array}$ & $\begin{array}{c}\text { Inner ring } \\
\text { failure }\end{array}$ & $\begin{array}{c}\text { Outer ring } \\
\text { failure }\end{array}$ & $\begin{array}{c}\text { Rolling element } \\
\text { failure }\end{array}$ & $\begin{array}{c}\text { Correct } \\
\text { rate }(\%)\end{array}$ \\
\hline BP & Drive end & $20 / 20$ & $18 / 20$ & $19 / 20$ & $20 / 20$ & 96.25 \\
GA-BP & Drive end & $20 / 20$ & $20 / 20$ & $20 / 20$ & $19 / 20$ & 98.75 \\
BP & Fan section & $20 / 20$ & $18 / 20$ & $19 / 20$ & $18 / 20$ & 93.75 \\
GA-BP & Fan section & $20 / 20$ & $20 / 20$ & $19 / 20$ & $19 / 20$ & 97.5 \\
\hline
\end{tabular}

The experimental results in Table 1 show that in terms of energy characteristic samples at the driving side and the fan side, the classification accuracy of the GA-BP neural network model is better than that of the BP neural network model. The experimental results also show that the GA is used to optimize the BP neural network, and the accuracy of rolling bearing fault diagnosis is verified.

\section{Conclusions}

Based on the research background of rolling bearing fault diagnosis, the GA-BP neural network is optimized and improved by wavelet packet decomposition and genetic algorithm according to the characteristics of the rolling bearing vibration signal. The bearing fault diagnosis model is used to carry out related experiments on the rolling bearing data set.

Experimental results show that the GA-BP neural network rolling bearing fault diagnosis model is adopted, and the accuracy and innovation are proved. The four-layer wavelet is used to remove the noise. The energy values of the eight reconstructed sub waveforms of the three-layer wavelet packet are taken as the eigenvalues of the denoised data. The subsequent classification algorithm can improve the calculation efficiency and the extracted energy features are normalized. Combined with GA-BP neural network, the wavelet packet decomposition method proposed achieves a good effect in the intelligent diagnosis of the rolling bearing fault. However, the method still has some shortcomings. Since there are many types of faults in rolling bearings, only a single fault in internal, external, and rolling elements of rolling bearing is diagnosed, and many mixed bearing fault problems cannot be detected and evaluated.

\section{Acknowledgment}

This work was supported by Academy-level scientific research projects of Guangzhou College of Technology and Business in 2019 (No. KA201929) and Industry-University Cooperation and Education Project of Ministry of Education in 2018 (No. 201801037086). 


\section{References}

[1] Lai C F, Chien W C, Yang L T, et al. (2019) LSTM and edge computing for big data feature recognition of industrial electrical equipment. IEEE Transactions on Industrial Informatics,2019, 15(4): 2469-2477.

[2] Deng W, Zhang S, Zhao $\mathrm{H}$, et al. A novel fault diagnosis method based on integrating empirical wavelet transform and fuzzy entropy for motor bearing. IEEE Access, 2018, 6: 35042-35056.

[3] Zhao H, Sun M, Deng W, et al. A new feature extraction method based on EEMD and multiscale fuzzy entropy for motor bearing. Entropy, 2017, 19(1): 14.

[4] Jian Y, Qing X, He L, et al. Fault diagnosis of motor bearing based on deep learning. Advances in Mechanical Engineering, 2019, 11(9): 1687814019875620.

[5] Wang X, Guo J, Lu S, et al. A computer-visionbased rotating speed estimation method for motor bearing fault diagnosis. Measurement Science and Technology, 2017, 28(6): 065012.

[6] Guo J, Shi B, Lei C, et al. Method of Compressed Sensing for Mechanical Vibration Signals Based on Double Sparse Dictionary Model. Journal of Mechanical Engineering, 2018, 54(6): 118.

[7] Ma X, Wang Y, Lu L, et al. Design of a $100-\mathrm{GHz}$ double-sideband low-IF CW Doppler radar transceiver for micrometer mechanical vibration and vital sign detection. IEEE Transactions on Microwave Theory and Techniques, 2020, 68(7): 2876-2890.

[8] Hoang D T, Kang H J. A motor current signalbased bearing fault diagnosis using deep learning and information fusion. IEEE Transactions on Instrumentation and Measurement, 2019, 69(6): 3325-3333.

[9] Wenliao D, Zhiqiang G, Xiaoyun G, et al. Multifractal characterization of mechanical vibration signals through improved empirical mode decomposition-based detrended fluctuation analysis. Proceedings of the Institution of Mechanical Engineers, Part C: Journal of Mechanical Engineering Science, 2017, 231(22): 4139-4149.

[10] He D, Cao H, Wang S, et al. Time-reassigned synchrosqueezing transform: The algorithm and its applications in mechanical signal processing. Mechanical Systems and Signal Processing, 2019, 117: 255-279.

[11] Li Z, Tao Y, Abu-Siada A, et al. A new vibration testing platform for electronic current transformers. IEEE Transactions on Instrumentation and Measurement, 2018, 68(3): 704-712.

[12] Gomes G F, Mendez Y A D, Alexandrino P S L, et al. A review of vibration based inverse methods for damage detection and identification in mechanical structures using optimization algorithms and ANN. Archives of computational methods in engineering, 2019, 26(4): 883-897.

[13] Li S, Wang J, Li X. Theoretical analysis of adaptive harmonic window and its application in frequency extraction of vibration signal. Journal of Central South University, 2018, 25(1): 241250.

[14] Aimer A F, Boudinar A H, Benouzza N, et al. Bearing fault diagnosis of a PWM inverter fedinduction motor using an improved short time Fourier transform. Journal of Electrical Engineering \& Technology, 2019, 14(3): 12011210.

[15] Jung J, Park Y, Lee S B, et al. Monitoring journalbearing faults: making use of motor current signature analysis for induction motors. IEEE Industry Applications Magazine, 2017, 23(4): 1221.

[16] Lee C Y, Huang K Y, Hsieh Y H, et al. Optimal Intrinsic Mode Function Based Detection of Motor Bearing Damages. Applied Sciences, 2019, 9(13): 2587.

[17] Nayana B R, Geethanjali P. Analysis of statistical time-domain features effectiveness in identification of bearing faults from vibration signal. IEEE Sensors Journal, 2017, 17(17): 56185625.

[18] Xue S, Howard I. Torsional vibration signal analysis as a diagnostic tool for planetary gear fault detection. Mechanical Systems and Signal Processing, 2018, 100: 706-728.

[19] Afrasiabi S, Mohammadi M, Afrasiabi M, et al. Modulated Gabor filter based deep convolutional network for electrical motor bearing fault classification and diagnosis. IET Science, Measurement \& Technology, 2021, 15(2): 154162.

[20] Lee G R, Gommers R, Waselewski F, et al. PyWavelets: A Python package for wavelet analysis. Journal of Open Source Software, 2019, 4(36): 1237.

[21] Xiao S, Lu Z, Wang P. Multivariate global sensitivity analysis for dynamic models based on wavelet analysis. Reliability Engineering \& System Safety, 2018, 170: 20-30.

[22] Hamid M, Usman M, Haq R U, et al. Wavelet analysis of stagnation point flow of nonNewtonian nanofluid. Applied Mathematics and Mechanics, 2019, 40(8): 1211-1226.

[23] Lee C Y, Cheng Y H. Motor Fault Detection Using Wavelet Transform and Improved PSO-BP Neural Network. Processes, 2020, 8(10): 1322.

[24] Li C, Xiong J, Zhu X, et al. Fault Diagnosis Method Based on Encoding Time Series and Convolutional Neural Network. IEEE Access, 2020, 8: 165232-165246.

[25] Liao Z, Song L, Chen P, et al. An automatic filtering method based on an improved genetic algorithm - with application to rolling bearing fault signal extraction. IEEE Sensors Journal, 2017, 17(19): 6340-6349. 